\title{
New method for measuring longitudinal fluctuations and directed flow in ultrarelativistic heavy ion reactions
}

\author{
L. P. Csernai, ${ }^{1}$ G. Eyyubova, ${ }^{2,3}$ and V. K. Magas ${ }^{4}$ \\ ${ }^{1}$ Institute of Physics and Technology, University of Bergen, Allegaten 55, 5007 Bergen, Norway \\ ${ }^{2}$ Department of Physics, University of Oslo, P.O. Box 1048 Blindern, 0316 Oslo, Norway \\ ${ }^{3}$ Skobeltsyn Institute of Nuclear Physics, Moscow State University, 199991 Moscow, Russia \\ ${ }^{4}$ Departament d'Estructura i Constituents de la Matèria, Universitat de Barcelona, 08028 Barcelona, Spain
}

(Received 22 June 2012; published 24 August 2012)

\begin{abstract}
It has been shown in recent ALICE@LHC measurements that the odd flow harmonics, in particular, a directed flow $v_{1}$, occurred to be weak and dominated by random fluctuations. In this work we propose a new method, which makes the measurements more sensitive to the flow patterns showing global collective symmetries. We demonstrate how the longitudinal center of mass rapidity fluctuations can be identified, and then the collective flow analysis can be performed in the event-by-event center of mass frame. Such a method can be very effective in separating the flow patterns originating from random fluctuations, and the flow patterns originating from the global symmetry of the initial state.
\end{abstract}

DOI: 10.1103/PhysRevC.86.024912

PACS number(s): 25.75.Ld, 25.75.Gz, 25.70.Pq

\section{INTRODUCTION}

Directed flow was the first and most dominant flow pattern in early relativistic heavy ion studies [1]. It provided a solid base for the subsequent more detailed works with higher multipole components. With increasing beam energy the magnitude of the directed flow decreased as the longitudinal momentum increased considerably, and this made the measurement of this flow component more difficult.

Recently the strong elliptic flow was demonstrated at the LHC, exceeding all measurements at lower energies for peripheral collisions [2]. At the same time recent measurements indicate weak directed flow, dominated by fluctuations [3].

Nevertheless the experience from early studies tells us that identification of this flow component is possible, and it can be separated from a strongly fluctuating background. Our goal in this paper is to propose a new method, which makes the analysis of the flow patterns to be more sensitive to global collective symmetries, by identifying and then correcting for the longitudinal center of mass (c.m.) rapidity $y^{\text {c.m. }}$ fluctuations. The proposed idea is rather general and can be implemented by all the experimental teams, although the particular way of identifying the c.m. rapidity event-by-event will depend on the particular setup.

Collective flow is parametrized by the azimuthal angle distribution around the beam axis via the expansion

$$
\begin{aligned}
\frac{d^{3} N}{d y d p_{t} d \phi}= & \frac{1}{2 \pi} \frac{d^{2} N}{d y d p_{t}}\left[1+2 v_{1}\left(y, p_{t}\right) \cos (\phi)\right. \\
& \left.+2 v_{2}\left(y, p_{t}\right) \cos (2 \phi)+\cdots\right],
\end{aligned}
$$

where $y$ is the rapidity, $p_{t}$ is the transverse momentum, and $\phi$ is the azimuthal angle in the transverse plane with respect to the impact parameter vector. The functions $v_{1}\left(y, p_{t}\right), v_{2}\left(y, p_{t}\right), \ldots$ are the harmonic flow expansion parameters.

Global collective flow patterns, which follow the global symmetries of the reaction event-by-event (EbE) provide valuable information of the overall pressure and transport properties of matter.

Random fluctuations, especially in the initial state, can also lead to flow processes; these however, are not correlated with the global collision geometry, and the correlation of the major axis of the asymmetry arising from the fluctuation may have no correlation with the reaction plane at all [4].

The goal of the present work is to identify and separate the global collective dynamical features from random fluctuations. We present some of the steps of such reconnaissance for global collective flow in high energy heavy ion reactions. This is particularly important for those harmonic components which are weak and difficult to identify.

The odd harmonics are dominated by fluctuating flow components which make it difficult to identify the weaker global collective flow components. Just as the transverse plane fluctuations, the beam directed fluctuations also modify the initial shape [5,6], the tilt of the longitudinal axis, and similarly the c.m. position. This issue is not discussed in the literature, although it influences all odd harmonics in the $v_{n}$ expansion.

Recent LHC data indicate that the reaction plane can be measured with forward and backward calorimeters where the projectile and target spectator residues are detected providing a reliable detection of the global event reaction plane (EP) [3] and the corresponding azimuthal angle $\Psi_{\mathrm{EP}}$.

To say something concrete, below we will mostly concentrate on the first flow component $v_{1}$, although, as it was already mentioned, the method is rather general and interesting for all odd components. According to a recent estimate [5] the $v_{1}$ flow shows a strong mirror antisymmetric structure as a function of rapidity, but this is smoothed out by random fluctuations of the c.m. motion of the participants. Furthermore it was pointed out that fluctuations may cause turbulence, i.e., random rotation [7]. Although, this was analyzed in the transverse plane, similar fluctuating rotation may also appear in the reaction plane, which may further soften the directed flow peak. 


\section{LONGITUDINAL CENTER OF MASS FLUCTUATIONS}

The idea is straightforward that if the participant $y^{\text {c.m. }}$ or $\eta^{\text {c.m. }}$ is strongly fluctuating, one can measure it experimentally and take the EbE c.m. into account when the odd flow components are evaluated. More than two dozen years ago a similar idea was developed to estimate $\mathrm{EbE}$ the azimuthal angle of the reaction plane better, and enable the directed flow analysis in low multiplicity and low acceptance reactions also [8].

If the acceptance cowers a large fraction of the momentum space of emitted particles, the initial c.m. of the system and the final measured c.m. are nearly identical. The final measured c.m. might deviate from the original one if a substantial part of the particles are not detected, especially if the notdetected particles are not evenly distributed in the momentum space.

\section{A. Participant rapidity from emitted particles}

The total four-momentum of all measured particles of one event is given by

$$
P=\sum_{\nu=1}^{M} p_{\nu}
$$

where $M$ is the measured multiplicity of the event. $P$ can be measured accurately for the pseudorapidity acceptance range of the detector, $|\eta| \leqslant \eta_{\max }$. The arising c.m. rapidity is

$$
y^{\text {c.m. }}=\frac{1}{2} \ln \frac{E+P_{z}}{E-P_{z}} .
$$

If we do not have a good mass resolution, the determination of $E_{i}$, and therefore of $E$, may become problematic, so only the pseudorapidity of the c.m. can be determined:

$$
\eta^{\mathrm{c} . \mathrm{m} .}=\frac{1}{2} \ln \frac{|\vec{P}|+P_{z}}{|\vec{P}|-P_{z}} .
$$

If the rapidity acceptance of the detector is limited, then the measured longitudinal single particle momenta are also constrained. On the other side, the transverse momenta are not constrained. For example at the ALICE Time-Projection Chamber (TPC) detector, the rapidity acceptance of the detector is limited to $|y|<0.9$, which means that the measured longitudinal nucleon momentum can not be more than $p_{\|}=$ $1 \mathrm{GeV} / c$. At the same time the nucleon transverse momentum distribution peaks at $p_{t}=1.2 \mathrm{GeV} / c$ [9], and a large part of the distribution extends to a few $\mathrm{GeV} / c$. Thus this constraint of the acceptance would result in underestimating the c.m. rapidity.

The pseudorapidity distribution for peripheral events was analyzed in a simple theoretical few source model [10] and for a detector with an acceptance of $|y|<0.9$, the reduction in c.m. pseudorapidity fluctuation was estimated to be $\left|\eta^{\text {c.m. }}\right|<0.2$. This is an estimate relevant for the ALICE TPC detector. In other detectors the acceptance might be wider; however, due to geometric limitations, such an underestimation of $\left|\eta^{\text {c.m. }}\right|$ may sill occur. Thus, we attempt to estimate $\eta^{\text {c.m. }}$ in another way.

\section{B. Participant rapidity from spectators}

Let us consider that we have three subsystems: (A) projectile spectators, (B) participants, and (C) target spectators. We can measure the energies of $\mathrm{A}$ and $\mathrm{C}, E_{A}$ and $E_{C}$, in the respective zero degree calorimeters (ZDCs): $\mathrm{ZDC}_{a}$ and $\mathrm{ZDC}_{c}$. Then the energy and momentum conservation gives

$$
\begin{aligned}
& E_{B}=A_{B} m_{B \perp} \cosh \left(y^{B}\right)=E_{\mathrm{tot}}-E_{A}-E_{C}, \\
& M_{B}=A_{B} m_{B \perp} \sinh \left(y^{B}\right)=-\left(M_{A}+M_{C}\right) .
\end{aligned}
$$

For example, at the present $\mathrm{LHC} \mathrm{Pb}+\mathrm{Pb}$ reaction with energy per nucleon $\epsilon_{N}=1.38 \mathrm{TeV} /$ nucleon, the beam rapidity is $y_{0}=7.986$ and

$$
E_{\text {tot }}=2 A_{P b} m_{N} \cosh \left(y_{0}\right),
$$

where $m_{N}=938.8 \mathrm{MeV} / \mathrm{c}^{2}$.

Furthermore the equations,

$$
\begin{aligned}
& E_{A}=A_{P} m_{N} \cosh \left(y_{0}\right), \\
& E_{C}=A_{T} m_{N} \cosh \left(-y_{0}\right),
\end{aligned}
$$

give the spectator numbers, $A_{P}$ and $A_{T}$, respectively, and

$$
\begin{aligned}
& M_{A}=A_{P} m_{N} \sinh \left(y_{0}\right), \\
& M_{C}=A_{T} m_{N} \sinh \left(-y_{0}\right),
\end{aligned}
$$

respectively, as well as the mass number of subsystem $B$ :

$$
A_{B}=2 A_{P b}-A_{P}-A_{T} .
$$

Thus for an event, dividing the second part of Eq. (5) by the first, we can determine the rapidity of subsystem $B$, which should be close to the rapidity of the participant system:

$$
y_{E}^{\text {c.m. }} \approx y^{B}=\operatorname{artanh}\left(\frac{M_{A}+M_{C}}{E_{\mathrm{tot}}-E_{A}-E_{C}}\right) .
$$

Our system B includes high energy "pre-equilibrium" particles, which are not detected by the ZDCs, and do not form a locally equilibrated system. To separate these two components from one another would need more information and a quantitative definition.

The part of the neutrons of the colliding nuclei, which are originating from the spectators and thus flying with beam rapidity (and energy), can be detected by the forward and backward neutron zero degree calorimeters. These ZDCs return the total energy of the measured neutrons. The ZDCs are between the two (projectile and target) beam pipes. The colliding nuclei propagate before and after passing through the point of intersection in these beam pipes. Magnetic fields deflect these and other charged fragments, so that only the uncharged neutrons end up in the ZDCs.

In central collisions the spectators contain very few, mainly single nucleons, and thus ZDC energies tend to zero on both sides, $\mathrm{A}$ and $\mathrm{C}$.

At higher impact parameters, the spectators will become more massive and the energy deposited in the ZDCs is increasing. At peripheral collisions, two residue spectators are expected at opposite sides of the participant zone. These may contain single protons and neutrons as well as bound nuclear fragments. Of these, only the single neutrons reach the ZDCs, 
because all charged fragments are deflected away from the joint beam direction (where the two colliding beams interact, and then are separated into the two beam pipes).

In the $\mathrm{LHC}, \mathrm{Pb}+\mathrm{Pb}$ reactions at $1.38 \mathrm{GeV} /$ nucleon beam energy in each beam result in a total neutron energy including both of the colliding $\mathrm{Pb}$ nuclei $E_{\text {tot }}^{n}=2 N_{P b} m_{N} \cosh \left(y_{0}\right)=$ $348 \mathrm{TeV}$, where $2 N_{P b}=2 \times 126$ is the total neutron number in the collision. The neutron ZDCs measure only single neutrons. At zero impact parameter we do not expect any spectators, while at the maximum impact parameter, $b=2 R_{P b}$, we do not expect any single neutrons, as the two spectators are two whole lead nuclei. At very small impact parameters the number of single neutrons are proportional to the number of spectators $N_{n}(b)=(N / A) N_{\text {Spect }}(b)$, where the nucleon number of the spectators in a collision at impact parameter $b$ can be obtained from the intersection geometry (like in the Glauber model). In the rest of the impact parameter range the spectators undergo nuclear multifragmentation, which is a well studied area both theoretically and experimentally [11]. These multifragmentation studies enable us to estimate the number of single neutrons for a given impact parameter $b$.

We can assume that at an intermediate impact parameter where the single neutron number of spectators is near to its maximum, we have less than half of the total neutron number of the spectators. Thus, we can assume the maximum of total ZDC energy of about $E_{\text {tot }}^{n}=160 \mathrm{TeV}$. In other words with this estimate of the $2 \times 126$ available neutrons, at most about $2 \times 58$ single neutrons can reach the ZDCs. With increasing impact parameter the number of bound nuclear fragments in the spectators increases, and thus these events are populating again the lower $E_{\mathrm{ZDC}}$ domains.

This feature can be checked by plotting $E_{\text {tot }}^{n}=E_{\mathrm{ZDCa}}+$ $E_{\mathrm{ZDCc}}$ as a function of the total charged multiplicity, $N_{\mathrm{ch}}$ measured, in the TPC. As discussed above the measured $E_{\text {tot }}^{n}$ first increases with decreasing $N_{\mathrm{ch}}$, then reaches a maximum at $N_{\text {ch }}^{\text {crit }} \approx 100$ to 200 , and then it decreases again. This enables us to separate the discussion of medium-peripheral collisions with multiplicity below $N_{\mathrm{ch}}^{\text {crit }}$ and extreme-peripheral collisions with multiplicity higher than $N_{\mathrm{ch}}^{\text {crit }}$. And thus we can separate the correlations between $E_{\mathrm{ZDCa}}$ and $E_{\mathrm{ZDCc}}$ for different multiplicity or centrality bins.

For example this feature of the ZDCs for the ALICE ZDCs is discussed in Ref. [12] in an early simulation, where incomplete spectator fragmentation is taken into account, and HIJING is used as event generator. For the estimates a model based on the ALADIN Collaboration Au-Au data is used, and deuterons are estimated from the NA49 Collaboration data. Based on the theoretical model estimates, the energy in the zero degree calorimeters versus the number of single spectator neutrons, initially raised monotonically up to about 100 neutrons and then it dropped rapidly. This rapid change was clearly attributed to the fact that at higher impact parameters the measured neutron number does not include neutrons in bound fragments, and thus the ZDC energy peaks at a critical neutron multiplicity [12].

The charged particle multiplicity does not provide a good reference measure for peripheral collisions, due to small multiplicities and therefore large fluctuations. As it is introduced in Ref. [2] we can have a better visualization of the spectator

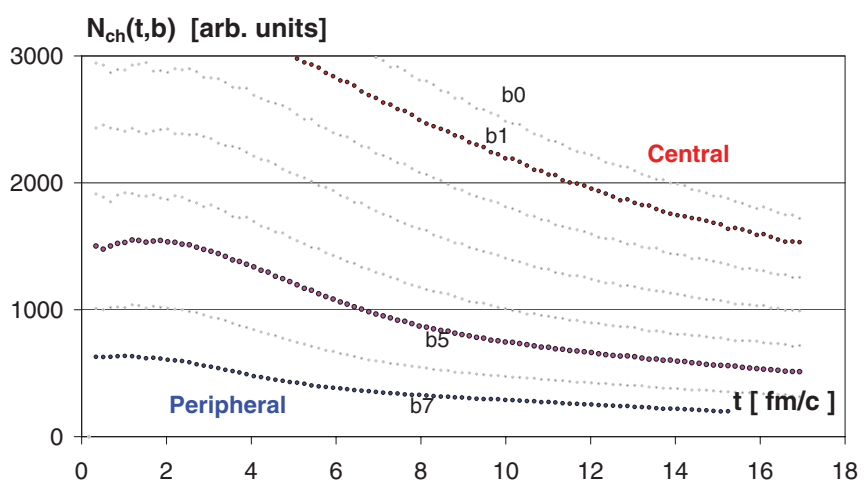

FIG. 1. (Color online) Simulation of the $\mathrm{Pb}+\mathrm{Pb}$ collision at the LHC with $\epsilon_{N}=1.38 \mathrm{TeV} /$ nucleon [5]. The calculated charged particle multiplicity $N_{\text {ch }}$ as a function of (FO) time (assuming a $t_{\mathrm{FO}}=$ const. FO hypersurface) for different impact parameters, $b=$ $0.0,0.1,0.2, \ldots, 0.7 b_{\max }$. The indicated $\left(b_{0}, b_{1}, \ldots, b_{7}\right)$ FO times for different impact parameters reproduce the measured charged particle multiplicities $N_{\mathrm{ch}}$ in the corresponding centrality bins. The visible fluctuations arise from the feature of the particle in cell (PIC) method [5], that the volume increases by one cell when a marker particle crosses the boundary. Thus at the initial state with relatively few cells and a large relative surface, this leads to fluctuations.

energy, if we plot the total ZDC energy, $E_{\mathrm{tot}}^{n}=E_{A}^{n}+E_{C}^{n}$, against the event centrality percentage.

The different presentations of these data are connected by the definition of centrality bins, which depend on the detector acceptance and the treatment of the lowest and highest multiplicities. The connection is presented in Fig. 1 of Ref. [2].

Now the above considerations and evaluation of $y_{\mathrm{c} . \mathrm{m}}$. should be reconsidered due to the fact that the ZDCs measure single neutrons only, and the number of single neutrons depends in a special way on the impact parameter due to the formation of nuclear fragments in the spectators or due to the incomplete dissociation of the bound spectator nuclear fragment in to single nucleons.

We can do a quantitative estimate of $y_{\text {c.m. }}$, if we conclude on the total spectator energy carried by all neutral and charged fragments together. Such a correction can be based on the measured $N_{\mathrm{ch}}$ and $N_{\mathrm{ch}}^{\text {crit }}$.

We may attribute the charged particle multiplicities or the centrality percentage to the obtained multiplicity estimated in a fluid dynamical (FD) model calculation, see Fig. 1, which is based on the calculations discussed in details in Ref. [5]. The impact parameters of the fluid dynamical model calculation can be matched well to the centrality percentages. The correspondence between impact parameter and event centrality percentage is impressive for semiperipheral reactions: $b=$ $0.2,0.3,0.4,0.5,0.6,0.7 b_{\max }$ correspond to the $5,13,21,29$, $37,45 \%$ centrality percentages, respectively.

We should correct the preliminary estimate, Eqs. (5) and (6), for the c.m. rapidity. We have to correct for the fact that nuclear fragments and the energy carried by these are not measured by the ZDCs, and thus our estimates for $E_{A}, E_{C}$, and thus $E_{B}$ should be modified and should be calculated based on the neutron energies measured in the ZDCs. 


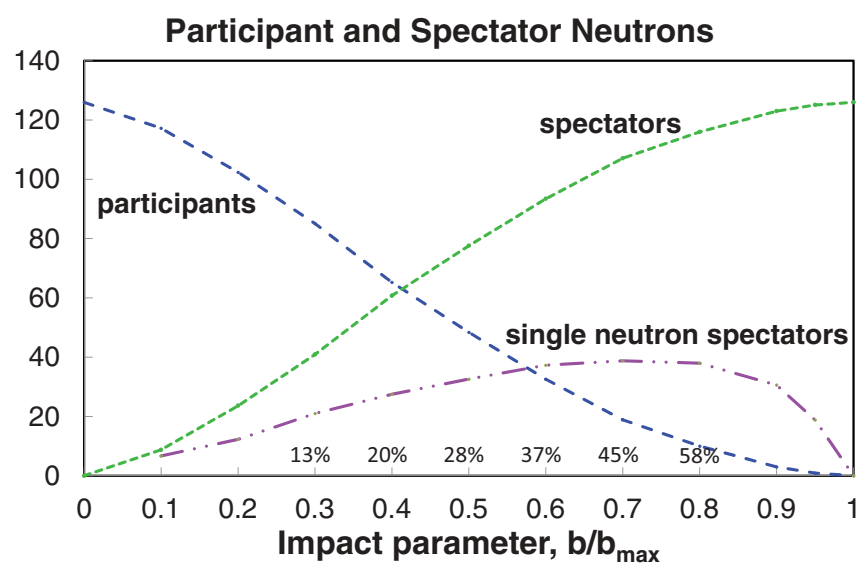

FIG. 2. (Color online) The number of participant neutrons from the projectile or from the target (dashed blue line) and the corresponding number of spectator neutrons (dotted green line) in one of the spectators (forward or backward) obtained in the initial state calculation [13] for a $\mathrm{Pb}+\mathrm{Pb}$ collision. The neutron distribution is assumed to be homogeneous in the system, $N / A=126 / 208$. At large impact parameters, part of the spectator neutrons remain in nuclear fragments, which are charged and so do not reach the neutron ZDCs. Based on the FD estimates, we relate the impact parameter with the centrality percentage, and based on nuclear multifragmentation studies [11] (see Table I), we estimate the number of single nucleons, which reach the neutron ZDCs (magenta dashed double dotted line).

\section{Neutrons in nuclear fragments}

The simplest approximation is that the total spectator energies, $E_{A}$ and $E_{C}$, are related the same way to the measured ZDC energies:

$$
E_{A}=(A / N) E_{A}^{n}, \quad E_{B}=(A / N) E_{B}^{n},
$$

where $A / N$ is the mass number to neutron number ratio in colliding nuclei (for simplicity we consider symmetric collisions). This approximation is satisfactory for central collisions and for small impact parameters $\left(b<0.3 b_{\max }\right)$, i.e. for relatively large charged multiplicities. Due to the presence of bound nuclear fragments in the spectators, we need a better approximation at intermediate or higher impact parameters.

Based on the initial state model [13], used in the three dimensional fluid dynamical calculations $[5,14]$, we get the number of participant nucleons for each impact parameter from the geometrical overlap of the colliding nuclei. On the projectile (or target) side 126/208 part of these are participant neutrons, $P_{n}(b)$, shown by the dashed blue line in Fig. 2 . The remaining neutrons are in spectators, shown by the dotted green line. As in Ref. [13], we assume that the $N / Z$ ratio is homogeneous in the whole initial state system.

Not all spectator neutrons are single neutrons; some of these are in composite nuclear fragments. The total energy of single neutron spectators are measured in the ZDCs, and thus their number can be easily obtained as the beam energy per nucleon is known. So, we can get the number of single neutrons, $N_{n}(b)$, as a function of the impact parameter $b$ in a straightforward way from the total measured ZDC neutron energy $E_{\mathrm{ZDCa}}+E_{\mathrm{ZDCc}}=E_{\mathrm{tot}}^{n}$ as a function of the impact parameter or the centrality percentage. The asymptotic behavior
TABLE I. The number of single spectator neutrons as function of centrality bins and the corresponding impact parameters as estimated based on the nuclear multifragmentation model [11]. The initial geometrical spectator numbers corresponding to a given impact parameter are also given.

\begin{tabular}{lccr}
\hline \hline$b / b_{\max }$ & Centrality $(\%)$ & $\left\langle N_{n}(b)\right\rangle$ & $P_{n}(b)$ \\
\hline & $0-10$ & 6.7 & 8.8 \\
$(0.2)$ & 6 & 12.3 & 23.5 \\
0.3 & $13 \pm 5$ & 21.0 & 40.9 \\
0.4 & $20 \pm 5$ & 27.5 & 60.8 \\
0.5 & $28 \pm 5$ & 32.6 & 77.6 \\
0.6 & $37 \pm 5$ & 37.3 & 93.4 \\
0.7 & $45 \pm 5$ & 38.8 & 107.1 \\
$(0.8)$ & $58 \pm 5$ & 38.0 & 116.0 \\
$(0.9)$ & $72 \pm 5$ & 30.6 & 123.0 \\
$(0.95)$ & $(84 \pm 5)$ & 18.8 & 124.6 \\
$(1.0)$ & $(90 \pm 5)$ & 0.0 & 126.0 \\
\hline \hline
\end{tabular}

of this dependence is not straightforward, $E_{\text {tot }}^{n} \longrightarrow 0$ both if $b / b_{\max } \longrightarrow 0$ or 1 . Based on nuclear multifragmentation studies mentioned above [11], we can estimate the number of single spectator neutrons, $N_{n}(b)$, as given in Table I. This estimate indicates that the number of detected single neutrons should have a maximum of about $30 \%$ at $\sim 50 \%$ centrality percentage.

The difference between the average and maximum for the single neutron numbers leads to an estimate of the systematic error of the method, for estimating the total spectator energy and momentum from the single neutrons.

Part of these spectator neutrons are inside composite nuclear fragments, especially at large impact parameters. These are charged, and due to the magnetic fields directing the beam, these do not reach the central neutron ZDCs nor the proton ZDCs. As discussed above this reduces the energy detected in the ZDCs to about 130 to $140 \mathrm{TeV}$, and the maximum numbers of detected single neutrons to about 47 to 51. This maximum appears at the centrality percentage of $\sim 50 \%$, which corresponds to $b=0.75 b_{\max }$ according to our fluid dynamical model estimates.

Thus we estimate number of single neutrons in the spectators for each impact parameter. These estimates have some systematic error due the unavoidable theoretical input, and at very central collisions as well as for extreme peripheral collisions (where there are no data and the multiplicity is so low that the fluid dynamical approximation is not applicable). The multifragmentation process may have a fluctuation itself, which may interfere with the $E_{A} E_{C}$ asymmetry, and lead to some systematic error.

In a given experimental setup one can measure directly $N_{n}(b)$, via measuring the average of the total energy in the two ZDCs as function of the impact parameter $b$, and dividing it by the beam energy per nucleon. This direct measurement of $N_{n}(b)$ should be preferred, as it eliminates a large part of the uncertainties of the theoretical approach arising from directly emitted high energy neutrons, and special emission evaporation mechanisms with large shear at the boundary between spectators and participants. 
Now we have to estimate, at a given centrality percentage (or impact parameter $b$ ), the total energy of the spectator residues (including protons and charged fragments), $E_{A}$ and $E_{C}$, from the energy of measured single neutrons in the ZDCs, $E_{A}^{\mathrm{sn}}$ and $E_{C}^{\mathrm{sn}}$. From the measured spectator neutron energies, $E_{A}^{\mathrm{sn}}$ and $E_{C}^{\mathrm{sn}}$, we get the corresponding single neutron numbers, $N_{A}^{\mathrm{sn}}=E_{A}^{\mathrm{sn}} / \epsilon_{N}$ and $N_{C}^{\mathrm{sn}}=E_{C}^{\mathrm{sn}} / \epsilon_{N}$. Multiplying these numbers by the correction factor, $P_{n}(b) / N_{n}(b)$, we get the spectator numbers, $A_{P, T}=N_{A, C}^{\text {sn }} P_{n}(b) / N_{n}(b)$, and spectator energies, including the contributions of single protons and of all nucleons bound in composite nuclear fragments:

$$
\begin{aligned}
& E_{A}(b)=(A / N) E_{A}^{\mathrm{sn}} P_{n}(b) / N_{n}(b), \\
& E_{C}(b)=(A / N) E_{C}^{\mathrm{sn}} P_{n}(b) / N_{n}(b) .
\end{aligned}
$$

This yields the corresponding spectator momenta, $M_{A}, M_{C}$, and we can get the event by event c.m. rapidity as in Eq. (6):

$$
y_{E}^{\text {c.m. }}(b) \approx y^{B}=\operatorname{artanh}\left(\frac{M_{A}+M_{C}}{E_{\text {tot }}-E_{A}-E_{C}}\right)-y^{\text {c.m. }}(b),
$$

where the last term is added to correct for eventual detector asymmetry, which is measurable, for all events of the sample for a given multiplicity percentage bin. Now, $E_{A}, E_{C}, A_{P}$, and $A_{T}$ are estimated and in the estimate we used the average $N_{n}(b)$, based on the estimated or eventually measured ZDC energies.

The correction increases for increasing impact parameter, which leads to increased estimates for $y_{\text {c.m. }}$ fluctuations; on the other hand the correction also increases and this leads to the possibilities of larger systematic errors.

At extreme peripheral reactions, spectators appear in the form of bound, and so charged, nuclear fragments, which are diverted by the magnetic field and are not detected. These provide more room for large c.m. rapidity fluctuation, but unfortunately neither the ALICE TPC nor the ZDCs can measure these reactions with the needed acceptance.

The above suggested analysis, by using the ZDC and charged multiplicity information together, may still provide the best estimate for the longitudinal fluctuations.

\section{Participant rapidity fluctuations}

In central collisions the ellipticity from random fluctuations can easily supersede the one from the global symmetry. This is indicated by the recent flow measurements, where the axes of different higher harmonics are uncorrelated, indicating that the source is random fluctuations, which are not correlated with the global reaction plane.

The global asymmetry may become dominant at higher impact parameters, i.e., $b>0.6$ to $0.8 b_{\max }$. For example, using the observed multiplicity in the ALICE TPC the centrality bins were defined as shown in Ref. [2]. These centrality bins can be assigned to impact parameter intervals, based on theoretical and geometrical model assumptions.

For example, in Ref. [5], simulations of the $\mathrm{Pb}+\mathrm{Pb}$ collisions at the LHC have been performed. The comparison of the multiplicity estimates of this model provide a correspondence between the impact parameter and the centrality percentage, see Fig. 1.
For the azimuthal flow asymmetries arising from random fluctuations, we cannot expect a correlation between the reaction plane (as measured by the spectator residues) and the asymmetry observed in the TPC. This also applies to the center of mass of the observed particles, because the longitudinal fluctuations are also not correlated with the spectator residues. Thus, we can expect a correlation mainly at peripheral collisions, where the ellipticity arising from global symmetry may be significant and may exceed the one caused by random fluctuations. Based on the above discussed correspondence between the multiplicity and the impact parameter, we can expect to see global effects at a $50 \%$ centrality bin or higher, i.e., in rather peripheral collisions.

The collective global flow components appear at moderate momenta; thus the c.m. determination should be sufficient for this component of the flow. In the previous subsection two methods were presented to estimate the c.m. rapidity. Using the TPC with the restricted in pseudorapidity acceptance limits the observed longitudinal momenta, while the transverse momenta are not constrained. Due to this the TPC is expected to underestimate the c.m. rapidity fluctuations.

The other method based on the single neutrons in the ZDCs is not constrained the same way. On the other hand one has to estimate the total spectator energy and momentum from the single neutrons, and this step requires some theoretical estimate which introduces a systematic error. This is illustrated by the fact that the correction factor, $P_{n}(b) / N_{n}(b)$, can be based on the average single neutron multiplicity. $N_{n}(b)$ can also be obtained from the theoretical estimates or the measured data, where the total single neutron energy, measured in the two ZDCs, is divided by the beam energy per single neutron, $\epsilon_{N}$.

The EbE data would show large fluctuations within each event among the emitted particles, exceeding the c.m. fluctuations considerably. We expect that compared to these fluctuations the c.m. fluctuations are small.

\section{Correlation between the TPC and ZDC c.m. rapidities}

Before we start to study the correlations we have to remove the average c.m. rapidity shift from the data as this arises from asymmetries in the detector acceptance. This is probably negligible for the TPC but expected to be significant for the ZDC data.

The azimuthal correlations of global collective flow are in correlation with the reaction plane; also the longitudinal c.m. rapidity should be globally defined for the flow pattern. In such a situation one would expect that the TPC and ZDC rapidities should strongly correlate.

On the other hand if the longitudinal and azimuthal correlations arise from random fluctuations, which are not correlated with the global symmetry axes, then such a correlation for c.m. rapidities measured at different rapidity ranges should not correlate.

We anticipate that at central and semicentral collisions there is no significant correlation or anticorrelation among the c.m. rapidities shown by the particles detected in the TPC or the ZDCs. In these collisions the correlations are arising dominantly from random fluctuations. 
For peripheral collisions of $50 \%$ centrality percentage or more, a weak positive correlation is anticipated. Here the global collective asymmetry (eccentricity) is so strong, that the global correlations start to become competitive.

\section{ADJUSTMENT OF THE CENTER OF MASS}

Substantial initial state rapidity fluctuations will average out all flow structures around the c.m. when the measurements are assuming that the c.m. is identical with the precollision c.m. of the given experiment (i.e., fixed to the laboratory frame in a colliding beam experiment with a symmetric, $\mathrm{A}+\mathrm{A}$, collision). Odd components of global collective flow patterns $\left(v_{1}, v_{3}, \ldots\right)$ are mirror asymmetric (MA) around the real participant c.m., so these are severely affected by the EbE c.m. fluctuations $[5,6]$.

Based on the above results, we suggest using the c.m. rapidity, EbE, determined from the $\mathrm{ZDC}$ data. Let us assume that the c.m. rapidity is measured for each event $E$ :

$$
y_{E}^{\text {c.m. }}(b)=\frac{1}{2} \ln \frac{E+P_{z}}{E-P_{z}},
$$

where $P=\sum_{\nu=1}^{M} p_{\nu}$ is the total measured four-momentum of event $E$. Here we consider that all corrections are taken into account as described in Eq. (8). Let us then shift each event to its own c.m. by the measured $y_{E}^{\text {c.m. }}$ so that each particle rapidity $y_{i}$ will be moved to

$$
y_{i}^{\prime}=y_{i}-y_{E}^{\mathrm{c} . \mathrm{m} .}(b) .
$$

This transformation will not affect the azimuthal angle of the emitted particles, $\vec{p}_{\perp, i}$, nor $m_{\perp, i}$; however, the longitudinal momentum and the energy will change to

$$
\begin{aligned}
p_{z, i}^{\prime} & =m_{\perp, i} \sinh \left(y_{i}-y_{E}^{\text {c.m. }}\right), \\
E_{i}^{\prime} & =m_{\perp, i} \cosh \left(y_{i}-y_{E}^{\text {c.m. }}\right) .
\end{aligned}
$$

Consequently the $\operatorname{EbE} v_{n}\left(p_{\perp}\right)$ will not change after adjusting the center of mass rapidity (if we integrate over the full rapidity range). Instead in a given event $E$, this adjustment will contribute to a redistribution of $v_{n}\left(y_{i}\right)$ to another set of rapidities, $y_{i}^{\prime}$. Consequently the detector acceptance boundaries will also change $\mathrm{EbE}$, and so one will have a continuous rapidity coverage even if the detectors have some rapidity acceptance gaps. Then, the flow harmonics can be determined by EbE averaging over all measured particles in the event:

$$
v_{n}\left(y^{\prime}, p_{\perp}\right)_{E}=\left\langle\cos \left[n\left(\phi_{i}-\Psi_{\mathrm{EP}}\right)\right]\right\rangle_{E},
$$

and then one can make an average over all events in a centrality bin:

$$
v_{n}\left(y^{\prime}, p_{\perp}\right)=\left\langle v_{n}\left(y^{\prime}, p_{\perp}\right)_{E}\right\rangle .
$$

If the centrality bin is wide, this two step averaging is essential [15].

As usual, one can also correct for fluctuations of the observed event plane azimuthal angle $\Psi_{\mathrm{EP}}$, compared to the real (precollision, but not measurable) reaction plane $\Psi_{R}$, as $\Psi_{R} \neq \Psi_{\mathrm{EP}}$, and so the observed $v_{n}^{\text {obs }}$ must be corrected by dividing by the resolution of the event plane [16].
In case of peripheral events with small multiplicity, one should also consider self-correlations in the determination of $y_{E}^{\text {c.m. }}(b)$, similarly to the correction of event plane determination in Ref. [8]. When we make the rapidity shift, Eq. (9), for a given particle $i$, then this particle may have also contributed to the determination of $y_{E}^{\text {c.m. }}(b)$, which then leads to a self-correlation. Note that in case of determining the rapidity shift from spectators and evaluating the flow harmonics from participants, this self-correlation does not happen. In other cases, just as in Ref. [8], we should evaluate the rapidity shift for a given particle in a way that that particle $i$ itself is left out from the calculation of the rapidity shift giving $y_{E-i}^{\text {c.m. }}(b)$ instead of $y_{E}^{\text {c.m. }}(b)$, and using the modified shift in Eq. (9).

So, using Eqs. (8) and (9) we can perform this rapidity shift event-by-event. To eliminate a relatively large rapidity binning of the sample, the events could be distributed uniformly and randomly inside each rapidity bin. Then the pseudorapidity distribution of $v_{1}$, as measured in the EbE c.m. frame, is expected to show a clear $\pm y$ asymmetry as the longitudinal, $y^{\text {c.m. }}$ fluctuations are removed from the data. The distribution this way may show a distinctly antisymmetric distribution in rapidity, as required by the global symmetry of the event.

In case of ALICE, for example, the TPC data are available in terms of pseudorapidity and integrated over the $p_{\perp}$ acceptance of the TPC. In such a situation instead of Eq. (9), we can use the approximation that the pseudorapidity, EbE, is shifted by the c.m. rapidity:

$$
\eta_{i}^{\prime}=\eta_{i}-y_{E}^{\mathrm{c} . \mathrm{m} .}(b) .
$$

So, this way the collective part of the flow may be identified.

We can quantify the identification of the collective symmetric versus the random fluctuating contribution to the flow the following way. We can evaluate the odd and even components of the flow [3]:

$$
\begin{aligned}
& v_{n}^{\text {odd }}\left(y^{\prime}, p_{\perp}\right)=\left[v_{n}\left(y^{\prime}, p_{\perp}\right)+v_{n}\left(-y^{\prime}, p_{\perp}\right)\right], \\
& v_{n}^{\text {even }}\left(y^{\prime}, p_{\perp}\right)=\left[v_{n}\left(y^{\prime}, p_{\perp}\right)-v_{n}\left(-y^{\prime}, p_{\perp}\right)\right] .
\end{aligned}
$$

We can use here also the approximation, Eq. (12), to evaluate the symmetries of the data.

Initial states with global collective symmetry are fully MA states, while fluctuating states may have both MA and mirror symmetric (MS) components. Then the two, odd and even components for $v_{1}\left(\eta^{\prime}\right)$ should show a distinct difference. The original $v_{1}\left(\eta^{\prime}\right)$ component is expected to be very close to the odd component, while the even component should be much smaller in the central rapidity range. This would indicate that $v_{1}\left(\eta^{\prime}\right)$ is MA to a large extent, and the MS component is consistent with zero.

\section{A. Prediction for central collisions}

At zero impact parameter global symmetry does not result in any global azimuthal asymmetry, so all harmonic coefficients must vanish from global collective origin and only random initial state fluctuations as well as dynamical random fluctuations developing during the collision may lead to azimuthal fluctuations. The recent ALICE analysis of 
azimuthal flow asymmetries in central collisions [3] could identify flow harmonics from $v_{1}$ to $v_{8}$, and shows a maximum for $v_{3}$ !

These data were evaluated in the laboratory c.m. frame, and in this frame longitudinal EbE fluctuations are also present. The new method presented here, by shifting the c.m. for every event to its own observed c.m., and evaluating the flow harmonics $v_{n}$ in that frame, is expected to test a spherically and azimuthally more symmetric system. This would result in a more symmetric azimuthal distribution, so the peak of the multipolarity distribution would move towards higher $v_{n}$ values. As a consequence the peak value, $v_{3}$, would decrease, while the value of $v_{4}$ would increase using this c.m. correction.

For central or almost central collisions the rapidity shift is small, so for these small rapidity shifts the use of ZDCs is not necessary, and one can determine the EbE rapidity shift directly from the data measured by the central TPC. This simplifies the method significantly. Furthermore, in central collisions the mass and the energy of the spectators are small, leading to large relative fluctuations. So, the determination of c.m. from the spectators would be inaccurate.

\section{CONCLUSIONS}

We presented a method how to analyze the directed flow data by considering the EbE longitudinal rapidity fluctuation of the participants. The participant c.m. rapidity can be estimated both from the TPC and the ZDC data. The TPC data are constrained to the pseudorapidity acceptance range, which impairs the c.m. rapidity estimate for peripheral reactions. To obtain an estimate for peripheral reactions from the ZDC data we estimate the forward and backward spectator energies from the measured single neutron energies. For peripheral reactions, where the azimuthal asymmetry of overall global collective origin is expected to exceed those, which originate from random initial state fluctuations, we expect to find significant correlation between the TPC and ZDC estimates of the c.m. rapidity of the participants, in the central rapidity range.

Using the ZDC data, which are not constrained by the limited acceptance, we describe each event in its own c.m. frame, and propose to evaluate the directed flow from these shifted data.

The method of shifting the system origin event by event to the rapidity of the participant c.m. rapidity is effective in separating the flow patterns originating from random fluctuations and the flow patterns originating from the global symmetry and asymmetry of the initial state.

\section{ACKNOWLEDGMENTS}

This work is supported in part by the European CommunityResearch Infrastructure Integrating Activity "Study of Strongly Interacting Matter" (HadronPhysics2, Grant Agreement No. 227431) under the Seventh Framework Programme of the EU, and the work of V.K.M. is partly supported by the Contract No. FIS2008-01661 from MICINN (Spain) and by the Generalitat de Catalunya Contract No. 2009SGR-1289. G.E. thanks colleagues at the University of Bergen, where part of this work was done, for the enlightening discussions and Norwegian State Educational Loan Fund for the financial support.
[1] H. Stöcker et al., Phys. Rev. C 25, 1873 (1982); G. Buchwald, L. P. Csernai, J. A. Maruhn, W. Greiner, and H. Stöcker, ibid. 24, 135 (1981); H. Stöcker et al., Phys. Rev. Lett. 47, 1807 (1981); L. P. Csernai, W. Greiner, H. Stöcker, I. Tanihata, S. Nagamiya, and J. Knoll, Phys. Rev. C 25, 2482 (1982); L. P. Csernai, P. Freier, J. Mevissen, H. Nguyen, and L. Waters, ibid. 34, 1270 (1986).

[2] K. Aamodt et al. (ALICE Collaboration), Phys. Rev. Lett. 105, 252302 (2010).

[3] K. Aamodt et al. (ALICE Collaboration), Phys. Lett. B 708, 249 (2012); I. Selyuzhenkov et al. (ALICE Collaboration), J. Phys. G 38, 124167 (2011); G. Eyyubova (ALICE Collaboration) (unpublished).

[4] A. Bilandzic et al. (ALICE Collaboration), J. Phys. G 38, 124052 (2011); M. Krzewicki et al. (ALICE Collaboration), ibid. 38, 124047 (2011).

[5] L. P. Csernai, V. K. Magas, H. Stöcker, and D. D. Strottman, Phys. Rev. C 84, 024914 (2011).
[6] Y. Cheng, Y. L. Yan, D. M. Zhou, X. Cai, B. H. Sa, and L. P. Csernai, Phys. Rev. C 84, 034911 (2011).

[7] S. Floerchinger and U. A. Wiedemann, J. High Energy Phys. 11 (2011) 100.

[8] P. Danielevicz and G. Odyniec, Phys. Lett. B 157, 146 (1985).

[9] J. Schukraft et al. (ALICE Collaboration), J. Phys. G 38, 124003 (2011).

[10] A. M. Skålvik (private communication and unpublished).

[11] P. R. Subramanian et al., J. Phys. G 7, L241 (1981); J. P. Bondorf et al., Phys. Rep. 257, 133 (1995); L. G. Moretto et al., ibid. 287, 249 (1997); V. E. Viola et al., ibid. 434, 1 (2006).

[12] M. Monteno et al. (ALICE Collaboration) (unpublished).

[13] V. K. Magas, L. P. Csernai, and D. D. Strottman, Phys. Rev. C 64, 014901 (2001); Nucl. Phys. A 712, 167 (2002).

[14] L. P. Csernai et al., Nucl. Phys. A 834, 261c (2010).

[15] D. M. Zhou et al., Eur. Phys. J. A 45, 353 (2010).

[16] A. M. Poskanzer and S. A. Voloshin, Phys. Rev. C 58, 1671 (1998). 